\title{
Influenza immunisation program at three tertiary paediatric hospitals in NSW in 2010
}

\author{
Nicholas J. Wood ${ }^{\mathrm{A}, \mathrm{B}}$ and Patrick M. Cashman ${ }^{\mathrm{C}, \mathrm{D}}$ \\ ${ }^{\mathrm{A}}$ National Centre for Immunisation Research \\ and Surveillance of Vaccine-Preventable Diseases \\ ${ }^{\mathrm{B}}$ The Children's Hospital at Westmead \\ ${ }^{\mathrm{C}}$ Population Health, Hunter New England Local Health District \\ ${ }^{\mathrm{D}}$ Corresponding author.Email: patrick.cashman@hnehealth. \\ nsw.gov.au
}

\begin{abstract}
This is a report of an innovative influenza immunisation program in three tertiary paediatric hospitals in NSW. A targeted once-off program of influenza immunisation funded by NSW Health was offered during 2010 at the Children's Hospital at Westmead, Sydney Children's Hospital and the John Hunter Children's Hospital. Authorised immunisers offered influenza immunisation to paediatric patients, outpatients and relatives of children with chronic illnesses. Influenza immunisation was administered to 3458 people, 1251 (36\%) of whom were children with chronic conditions. In 2009 before the program, 420 influenza vaccines were prescribed for children in two of these hospitals. This number increased to 949 in 2010, the year of the program. Dedicated vaccination clinics at tertiary paediatric hospitals provide additional opportunities to ensure that children at high risk of severe influenza disease and its complications are vaccinated. The information obtained from the hospital vaccination program contributed to the national investigation of febrile convulsions following influenza vaccines in children in 2010 .
\end{abstract}

Young children and infants have a high incidence of influenza infection, and hospitalisation for the treatment of influenza and its complications is most common in children less than 2 years of age. ${ }^{1-3}$ The Australian Immunisation Handbook recommends influenza immunisation for children over 6 months of age, and strongly recommends influenza immunisation for children over 6 months of age with medical conditions predisposing to severe influenza. ${ }^{4}$
Many children with moderate-to-severe chronic medical conditions attend outpatient care at one of the three major paediatric hospitals in New South Wales (NSW), the Children's Hospital at Westmead, Sydney Children's Hospital and the John Hunter Children's Hospital. Over 400000 children are seen each year in the outpatient departments at the three hospitals; approximately 290000 children are treated at the Children's Hospital at Westmead, 100000 at Sydney Children's Hospital and 20000 at the John Hunter Children's Hospital. ${ }^{5}$ The Sydney Children's Hospitals Network, including Sydney Children's Hospital and the Children's Hospital at Westmead, has over 44500 inpatient admissions annually, making it the largest Australian and New Zealand children's health service.

In 2010 NSW Health provided funding to conduct influenza vaccination at these three hospitals through a targeted once-off program. The program funding was for a 6-month period and was principally used to employ nurse immunisers with the aim of offering influenza immunisation to protect paediatric inpatients and outpatients and decrease the numbers of preventable influenza hospitalisations.

Siblings and parents of children with medical conditions attending outpatient clinics were also offered influenza vaccination to reduce opportunities of influenza exposure thereby cocooning the medically at-risk children.

This paper describes the program delivery of influenza vaccine to children with chronic medical conditions and their families at the three major paediatric hospitals in NSW in 2010.

\section{Methods}

The clinics at each hospital varied in location, timing and staffing. Individual details including unique hospital identifier, age, sex, presence of chronic medical condition, influenza vaccine received and contact details were recorded at the time of vaccination. All clinics commenced offering the monovalent H1N1 2009 vaccine, Panvax ${ }^{\circledR}$ (CSL Biotherapies, Australia), in February 2010 and changed to seasonal trivalent inactivated influenza virus (TIV) vaccine in March 2010 when it became available. The John Hunter Children's Hospital used only Influvac ${ }^{\circledR}$ (Solvay Biologicals, $0.5 \mathrm{~mL}$ ) as the seasonal influenza vaccine, as it was the only vaccine obtained by the hospital, while Sydney Children's Hospital and The Children's Hospital at Westmead used either Fluvax ${ }^{\circledR}$ 
Table 1. Numbers of influenza vaccines administered at three NSW paediatric hospitals to children with chronic medical conditions and their families, 2010

\begin{tabular}{|c|c|c|c|c|c|}
\hline & $\begin{array}{c}\text { Monovalent } \\
\text { H1N1 } \\
\text { (Panvax) } \\
n\end{array}$ & $\begin{array}{c}\text { Children with chronic } \\
\text { medical conditions given } \\
\text { monovalent H1N1 (Panvax) } \\
n(\%)\end{array}$ & $\begin{array}{l}\text { Seasonal } \\
\text { Influenza } \\
\text { vaccine } \\
n\end{array}$ & $\begin{array}{c}\text { Children with chronic } \\
\text { medical conditions given } \\
\text { seasonal influenza vaccine } \\
n(\%)\end{array}$ & $\begin{array}{l}\text { Total } \\
\text { administered } \\
\text { vaccine } \\
\mathrm{N}\end{array}$ \\
\hline $\mathrm{CHW}$ & 543 & $174(32)$ & 1427 & 413 (29) & 1970 \\
\hline $\mathrm{SCH}$ & 232 & $108(47)$ & 297 & $194(65)$ & 529 \\
\hline $\mathrm{JHCH}$ & 391 & $166(42)$ & 568 & $196(35)$ & 959 \\
\hline Total & 1166 & $448(38)$ & 2292 & $803(35)$ & 3458 \\
\hline
\end{tabular}

(CSL Biotherapies, $0.5 \mathrm{~mL}$ ), Fluvax $^{\circledR}$ junior (CSL Biotherapies, $0.25 \mathrm{~mL}$ ) or Influvac ${ }^{\circledR}$ (Solvay Biologicals, $0.5 \mathrm{~mL})$.

The clinics were advertised by posters around the hospital, through presentations at medical team meetings, during grand rounds and by individual approaches from the nurse immuniser to parents of patients attending the hospital. At the Children's Hospital at Westmead, nurse immunisers from the Sydney West Area Health Service (SWAHS) public health unit were seconded for the duration of the influenza vaccine program.

As this was a new approach in NSW, participating hospitals and their associated public health unit met monthly by teleconference to implement, coordinate and manage the program. These groups have continued to meet during the 2011 influenza season to coordinate and promote influenza immunisation and monitor vaccine safety.

We evaluated clinic outcomes using the following indicators: the number of children and members of their families immunised; a comparison of current vaccine administration against previous attempts to increase influenza vaccine coverage at the paediatric hospitals.

\section{National adverse events signal}

On Friday 23 April 2010, following the advice of the Chief Medical Officer of Australia, seasonal influenza vaccine administration to children aged 5 years and under was suspended due to an adverse events signal of possible increased fever reactions and febrile convulsions following influenza immunisation in young children. This significantly affected the influenza vaccine program in the three paediatric tertiary hospitals but clinic records provided a unique cohort of children to rapidly provide additional data to inform the national investigation.

From 27 April 2010, research nurses and clinic staff from the Children's Hospital at Westmead and the National Centre for Immunisation Research and Surveillance (NCIRS) used a scripted telephone interview to ask parents of children 6 months to 5 years old, who had received influenza vaccines in the three hospitals since the initiation of the program, about reactions following the receipt of the influenza vaccine. Parental reports were collated for each child who was vaccinated, and analysed by type of influenza vaccine received, age of the child, and the presence of chronic co-existing medical conditions (including gastroenterological, respiratory, oncological, cardiac and neurological conditions).

We therefore also evaluated the utility of the clinic in responding to and understanding a national adverse events signal.

\section{Results}

The combined number of influenza-containing vaccines (monovalent $\mathrm{H} 1 \mathrm{~N} 1$ or seasonal trivalent) given to children presenting with medical conditions and their families at the three hospitals was 3458 vaccines (Table 1). Children with chronic medical conditions accounted for $36 \%(n=1251)$ of vaccines administered.

The immunisation nurses reported that the offer to immunise family members to assist in the protection of their unwell child against influenza was popular and over 2000 family members were immunised.

The 2010 influenza program at the paediatric tertiary hospitals administered more influenza vaccinations to outpatients than in previous years (as measured by the influenza vaccines dispensed from the two hospital pharmacies that had provided ad hoc vaccination to paediatric outpatients previously) (Figure 1).

\section{National adverse events signal}

Commencing on 27 April 2010, parents of children 6 months to 5 years old $(n=333)$, who had received influenza vaccines in the three hospitals since the initiation of the program, were contacted. Overall nearly 20\% $(n=66)$ of parents reported that their child had a fever within 48 hours of vaccination. There was a statistically 


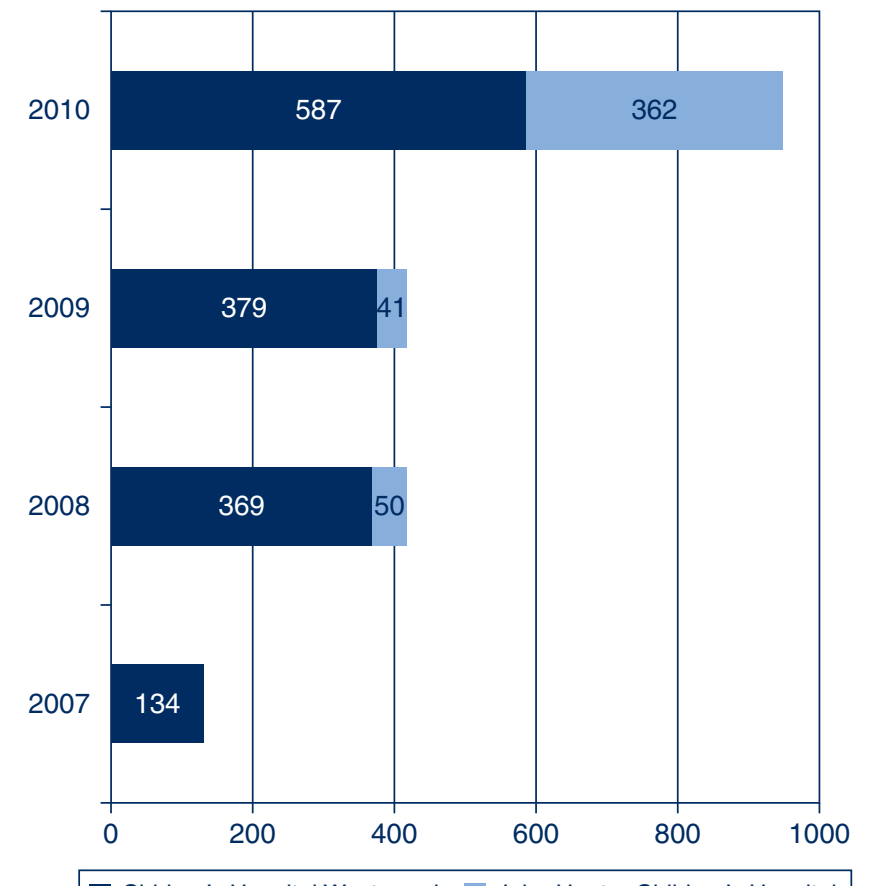

Chidren's Hospital Westmead John Hunter Children's Hospital

Figure 1. Influenza vaccines dispensed at two NSW paediatric hospitals: comparison of program year 2010 with previous years, 2007-2009.

Source: Hospital pharmacy records at The Children's Hospital at Westmead and the John Hunter Children's Hospital.

significant higher parental fever report for CSL Fluvax ${ }^{\circledR}$ compared with Influvac $^{\circledR}$ (RR 6.5, 95\% CI 3.1 to 13.9 , $p<0.0001$ ), or CSL Panvax ${ }^{\circledR}$ (RR 2.9, 95\% CI 1.8 to 4.3 , $p \leq 0.0001){ }^{6}$ There were no reports of febrile convulsions in any children.

\section{Discussion}

The dedicated paediatric hospital influenza vaccine program in 2010 immunised higher numbers of high medical at-risk children compared to previous years. Cocooning protection was offered through vaccination of family members. Having a database of the cohort of vaccinees allowed the hospitals to rapidly investigate an important safety alert and contribute to the national investigation of febrile convulsions following influenza vaccines in children in 2010 .

The Royal Children's Hospital, Melbourne, Victoria has had a dedicated immunisation service for many years, delivering vaccinations including annual influenza vaccine, providing immunisation advice and managing adverse events. Parents and siblings of patients are able to purchase influenza vaccine from the hospital pharmacy. This service delivered approximately 2000 doses of influenza vaccine to patients, parents and siblings in $2009 .^{7}$ Additional benefits of these hospital-based programs include the opportunity for specialists to seek advice regarding immunisation for their patients with complex medical conditions, the development of common vaccination protocols for use in complex medical cases, the capacity to investigate safety concerns quickly, and a training opportunity for hospital nursing staff with vaccines which are usually administered in primary care settings.

As the Australian Immunisation Handbook notes: 'it is vital that healthcare professionals take every available opportunity to vaccinate children and adults'. 4 It is particularly important that children with chronic medical conditions who are most at risk of severe vaccinepreventable diseases are vaccinated in a timely way.

\section{Conclusion}

Dedicated vaccination clinics at tertiary paediatric hospitals provide a valuable method of ensuring that children at high risk of severe influenza disease and its complications are vaccinated. The clinics can provide these vulnerable children with additional protection by vaccinating their family members (cocooning), while at the same time facilitate important clinical, surveillance and education activities.

\section{Acknowledgments}

NSW Health provided funding for the program in each of the paediatric hospitals. We thank the staff at each clinic site for their contribution.

\section{References}

1. Izurieta HS, Thompson WW, Kramarz P, Shay DK, Davis RL, DeStefano $\mathrm{F}$ et al. Influenza and the rates of hospitalisation for respiratory disease among infants and young children. $N$ Engl J Med 2000; 342: 232-9. doi:10.1056/NEJM200001273420402

2. Neuzil KM, Mellen BG, Wright PF, Mitchel EF, Jr, Griffin MR. Effect of influenza on hospitalization, outpatient visits and courses of antibiotics in children. $N$ Engl J Med 2000; 342: 225-31. doi:10.1056/NEJM200001273420401

3. Isaacs D. Should all Australian children be vaccinated against influenza? Med J Aust 2005; 182: 553-4.

4. National Health and Medical Research Council. The Australian immunisation handbook 9th edition, 2008. Canberra: Australian Government Publishing Service; 2008.

5. Australian Institute of Health and Welfare. Information on hospitals throughout Australia and how they compare against national and state and territory data. [http://www.aihw.gov.au/ australias-hospitals-at-a-glance-2009-10/] Available at: www.myhospitals.gov.au (Cited 15 September 2011).

6. Wood N, Sheppeard V, Cashman P, Palasanthiran P, Casacelli M, Cannings $\mathrm{K}$ et al. Influenza vaccine safety in children less than 5 years old. The 2010 and 2011 experience in Australia. Ped Inf Dis J. 2011 Nov 16. [Epub ahead of print]. doi:10.1097/ INF.0B013E31823D5303

7. 2010 Victorian public healthcare awards: A hospital based immunisation service. Available at: http://www.rch. org.au/emplibrary/rch_awards/VPHA2010_Final_ immunisationaward.pdf (Cited 15 September 2011). 